\title{
Preparation, Characterization and in vitro/in vivo Evaluation of Lovastatin-Loaded PLGA Microspheres by Local Administration for Femoral Head Necrosis
}

\author{
This article was published in the following Dove Press journal: \\ Drug Design, Development and Therapy
}

\section{Yang Sun \\ Di Long}

Department of Orthopaedics, Affiliated Central Hospital of Shenyang Medical College, Shenyang, People's Republic of China
Correspondence: Yang Sun Email sunyang797979@I63.com
Background: The present work is an effort to develop a novel locally injection LVTTloaded PLGA microspheres (LVTT-PLGA-MS) on the treatment of rabbits with femoral head necrosis (FHN).

Methods: LVTT-loaded PLGA microspheres (LVTT-PLGA MS) were prepared by an emulsion-solvent evaporation method. The physicochemical properties of LVTT-PLGA-MS were investigated to ensure that they have good qualities and are suitable for local delivery. In vitro drug release behavior of MS was also studied compared with free LVTT. In vivo, we also studied the pharmacokinetics and pharmacodynamics of MS in rabbits with the optimized formulation.

Results: In this study, we used the emulsion-solvent evaporation method to prepare LVTTPLGA MS. Scanning electron microscopy demonstrated that the LVTT-PLGA MS were regular, round in shape and relatively unified size distributions were selected. The mean PS was $12.3 \pm 2.1 \mu \mathrm{m}$. The drug-loading rate $(27.6 \% \pm 2.9 \%)$ was calculated for three batches of MS. The thermogram of LVTT-PLGA MS showed an endothermic peak at $98.3^{\circ} \mathrm{C}$, revealing that LVTT existed in MS in an uncrystallized rather than a crystallized form. In the release study, LVTT-PLGA MS is observed linear prolonging drug release rates for more than 21 days without initial burst release. The pharmacodynamic results confirmed that the LVTTPLGA MS had a good and lasting improvement effect against femoral head necrosis.

Conclusion: Our results demonstrated that LVTT-PLGA MS has the potential for being a local delivery system.

Keywords: lovastatin, PLGA, microspheres, in vitro/in vivo

\section{Introduction}

Statins are competitive 3-hydroxy-3-methyl coenzyme A (HMG CoA) reductase inhibitors, which are widely used in the treatment of hyperlipidemia and hypercholesterolemia. Previous studies showed that statins including lovastatin (LVTT) can induce bone marrow mesenchymal stem cells to differentiate into osteoblasts and promote the osteogenic ability of human or animal osteoblasts. ${ }^{1-3}$ In addition, statins have been shown to enhance angiogenesis by upregulating gene expression of vascular endothelial growth factor (VEGF) and basic fibroblast growth factor (FGF-2). ${ }^{4-6}$ Both VEGF and FGF-2 have been shown to induce bone morphogenetic protein-2 (BMP-2) expression and stimulate osteoblast differentiation indirectly. ${ }^{7,8}$ Yoshii et al reported that local injection of LVTT in biodegradable polyurethane scaffolds enhances bone regeneration in a critical-sized segmental 
defect in rat femora. ${ }^{1}$ It seems that a single administration of LVTT may enhance new bone formation by the two different pathways of osteogenesis and angiogenesis.

Since LVTT (with a water solubility of $0.4 \times 10-3 \mathrm{mg}$ / $\mathrm{mL}$ ) is considered to be an ideal intestinal lymphatic transport substrate due to its higher log P-values (4.3) and good oil solubility (38 and $42 \mathrm{mg} / \mathrm{mL}$ in carbitol and monoglycol, respectively). In addition, LVTT is rapidly metabolized in the intestine and liver, resulting in low oral bioavailability $(\sim 5 \%){ }^{9}$ Cytochrome P450 3A4 metabolizes the lactone form of LVTT into hydroxy acid and their metabolites. ${ }^{10,11}$ The availability of the drug to the systemic circulation is low and variable due to the extensive extraction of LVTT from the liver. ${ }^{12}$ In order to avoid the influence of individual differences and prevent rapid drug clearance, the use of a sustained-release drugdelivery system is a feasible strategy.

As a drug sustained-release system commonly used in clinical practice, polylactic-co-glycolic acid (PLGA) can specifically administer drugs, improve drug stability, and control drug release, so it has been applied in the treatment of many diseases. ${ }^{13,14}$ It was reported by scholars that there is a new application of LVTT in the form of PLGA microspheres (MS). ${ }^{15}$ However, its purpose was to improve oral bioavailability.

Thus, the present work is an effort to develop a novel locally injection LVTT-PLGA-MS on the treatment of rabbits with femoral head necrosis (FHN). LVTT-loaded PLGA microspheres (LVTT-PLGA MS) were prepared via an improved emulsion-solvent evaporation method. The physicochemical properties of LVTT-PLGA-MS were studied to ensure that LVTT-PLGA-MS had good quality and was suitable for local administration. The in vitro release behavior of MS and free LVTT was also studied. In vivo, we also studied the pharmacokinetics and pharmacodynamics of MS in rabbits with the optimized formulation.

\section{Materials and Methods}

\section{Materials}

Lovastatin (LVTT) was purchased from SuPuEr Chemical Co., Ltd. (Wuhan, China). Poly(L-lactide-co-glycolide) (PLGA 50:50) was a gift from the lab of Yacoo technology (Suzhou, China). Poly(vinyl alcohol) (PVA) was obtained Sinopharm Chemical Reagent (Shanghai, China). 4,5-(dimethylthiazol-2-yl) 2,5-diphenyl-tetrazolium bromide (MTT) was purchased from Sigma-Aldrich
(St. Louis, MO, USA). All other chemicals were of analytical grade. Distilled water was used in all experiments.

\section{Animals}

New Zealand White rabbits $(2.0-3.0 \mathrm{~kg}$, male) were provided by the Animal Experimental Center of Shenyang Medical College. The experimental animals were individually housed in an air-conditioned and lightcontrolled room at $24 \pm 1{ }^{\circ} \mathrm{C}$ and $65 \pm 5 \%$ relative humidity. They were given a standard pellet diet and were provided with water ad libitum. All animals were healthy and free of clinically observable ocular abnormalities. All animal experiments were performed in accordance with institutional guidelines, following a protocol approved by the Ethics Committees of Shenyang Medical College (ZGYKDX20201102). The guide of the National Institutes of Health for the care and use of laboratory animals was strictly followed.

\section{Preparation of LVTT-Loaded PLGA MS}

LVTT-loaded PLGA MS were prepared by the oil-inwater $(\mathrm{O} / \mathrm{W})$ emulsion/solvent evaporation method. ${ }^{15}$ In brief, $10 \mathrm{mg}$ LVTT and $300 \mathrm{mg}$ PLGA were dissolved in $2 \mathrm{~mL}$ of methylene chloride; this solution was dropped into $200 \mathrm{~mL}$ of $0.25 \%(\mathrm{w} / \mathrm{v})$ PVA solution at room temperature under a magnetic stirrer for $45 \mathrm{~min}$ until most of the methylene chloride evaporated, leaving nothing but solid MS. The microsphere suspension was centrifuged at $3000 \mathrm{rpm}$ for $15 \mathrm{~min}$ and the microspheres were washed 3 times with distilled water. After the organic solution was removed, the mixture was frozen and dried for $48 \mathrm{~h}$.

\section{Characterization}

Particle Size and Scanning Electron Microscopy (SEM) The intensity-mean particle size of MS was determined using a Zetasizer NanoZS (Malvern Instruments Ltd.). All measurements were performed under automatic mode at $25^{\circ} \mathrm{C}$. Software provided by the manufacturer was used to calculate the mean particle size (PS) of NGs. All measurements were performed at least in triplicate to calculate the mean values $\pm \mathrm{SD}$. The prepared MS were coated with a thin layer of gold by sputtering and then the microstructure was observed in a scanning electron microscope (SEM; AIS-2100 780, Seron, South Korea) that operated at an acceleration voltage of $20 \mathrm{kV}$. 


\section{Encapsulation Efficiency and Drug Loading}

Firstly, LVTT were extracted from MS with $1 \mathrm{~mL}$ ethanol and chloroform (10:90), and the extracted solution was then properly diluted prior to HPLC analysis (Agilent 1100 series). The relevant calculation formula is as follows: Encapsulation efficiency $\%=$ the total amount of simvastatin/the total amount of microspheres x $100 \%$. Drug loading $\%$ $=$ actual drug loading/theoretical drug loading $\times 100 \%$.

\section{Differential Scanning Calorimetry (DSC) Analysis}

DSC analysis was performed by DSC 8000 differential scanning calorimeter (Mettler-Toledo International Inc, USA). The accurately weighed samples were placed in an aluminum plate and sealed with a cover. $\mathrm{Al}_{2} \mathrm{O}_{3}$ was used as the reference material. During the scanning process, a heating rate of $5^{\circ} \mathrm{C} / \mathrm{min}$ was applied with the temperature range from $20^{\circ} \mathrm{C}$ to $150^{\circ} \mathrm{C}$ with the gas of $\mathrm{N}_{2}$.

\section{X-Ray Diffraction (XRD) Analysis}

XRD patterns were obtained at room temperature using a very high-resolution $\mathrm{Cu}-\mathrm{K}_{\alpha}$ radiation diffraction system operating at a voltage of $40 \mathrm{kV}$ and a current of $30 \mathrm{~mA}$. MS were analyzed in the $2 \theta$ angle range of $0-80^{\circ}$.

\section{Fourier Transform Infrared Spectroscopy (FTIR) Analysis}

Infrared spectroscopy was carried out to determine the chemical composition of the prepared MS using FTIR (Nicolet, USA) operating in the wavenumber range of $0-4000 \mathrm{~cm}^{-1}$ at the absorption mode.

\section{In vitro Release Studies}

In vitro release studies were performed using the USP basket apparatus (RC-806, TDTF, China). MS containing LVTT equivalent to $20 \mathrm{mg}$ was added to $300 \mathrm{~mL}$ of the dissolution medium ( $\mathrm{pH} 7.4, \mathrm{PBS}$ ), thermostated at $37 \pm 0.5^{\circ} \mathrm{C}$, and stirred at $100 \mathrm{rpm}$. At the intervals of 1, 3, 5, 7, 9, 12, 15, 18, $21 \mathrm{~d}$, $2 \mathrm{~mL}$ samples were withdrawn from the dissolution vessels and immediately replaced with the same volume of the fresh dissolution medium. Each sample was centrifuged for $5 \mathrm{~min}$ at $8000 \mathrm{rpm}$ and the supernatant was assayed by HPLC. The same doses of free LVTT were used as controls to participate in this experiment. Each experiment was repeated three times.

\section{Stability Study}

LVTT-loaded PLGA MS were packed in a glass vial and stored at various temperatures $\left(40 \pm 2^{\circ} \mathrm{C}\right.$ and $\left.5 \pm 2^{\circ} \mathrm{C}\right)$ for a period of 60 days. Sampling was done at predetermined time intervals of 0,30 , and 60 days. Estimation of drug content and dissolution study of these samples were performed. To compare the similarity of drug release profiles before and after stability studies, a statistical tool was used to determine the difference factor (f1) and the similarity factor (f2). ${ }^{16}$ The $f 2$ value ranging from 50 to 100 ensures the similarity of the two curves.

\section{HPLC Analysis}

The concentration of LVTT in the above sample was determined by high-performance liquid chromatography (HPLC). The separation was carried out at $30^{\circ} \mathrm{C}$ using a reverse-phase thermo $\mathrm{C} 18$ column $(5 \mu \mathrm{m}, 4.6 \mathrm{~mm} \times$ $250 \mathrm{~mm}$ ). The mobile phase consisted of acetonitrile and $0.01 \%$ phosphonic acid (60:40). The detection wavelength was $238 \mathrm{~nm}$ and a flow rate of $1.0 \mathrm{~mL} /$ minute was employed. A sample volume of $20 \mu \mathrm{L}$ was injected.

\section{Pharmacodynamic Evaluation Animals Modeling ${ }^{17}$}

After 1 week of adaptive feeding, $10 \mathrm{~mL} / \mathrm{kg}$ horse serum was injected intravenously at the ear edge of rabbits and the same horse serum was injected again 3 weeks later with the dose changed to $6 \mathrm{~mL} / \mathrm{kg}$; at a time interval of 2 weeks, methylprednisolone sodium succinate $45 \mathrm{mg} / \mathrm{kg}$ was injected into the same site, once daily for 3 days. Since methylprednisolone sodium succinate injections started, each rabbit had been intraperitoneally injected with penicillin 10 million units/day for 7 consecutive days; at the last injection, 2 rabbits were sacrificed randomly, and the femoral head specimens were fixed with $4 \%$ polyformaldehyde, decalcified, embedded in paraffin and stained with routine HE. Rabbit modeling was observed by histological methods and the criteria for successful modeling in each rabbit were avascular necrosis (AVN) of the femoral head. The modeling rabbits were randomly divided into five groups. After successful modeling, the method of administration was a local injection of the femoral

Table I The Group Information of the Pharmacodynamic Evaluation. The Method of Administration was Local Injection of Femoral Head with $5 \mathrm{mg} / \mathrm{kg}$ LVTT

\begin{tabular}{|l|l|l|}
\hline Group & Formulation & Animal Number \\
\hline 1 & Normal group & 6 \\
2 & Blank control group & 6 \\
3 & Core decompression therapy & 6 \\
4 & LVTT injection & 6 \\
5 & LVTT-PLGA MS & 6 \\
\hline
\end{tabular}

Note: Core decompression therapy means: Bone cement was infused into the unilateral femoral bone marrow cavity of rabbits to destroy the internal circulation of femoral bone marrow. 
head with $5 \mathrm{mg} / \mathrm{kg}$ LVTT for 1 week. The group information is shown in Table 1.

\section{Biochemical Indexes}

Blood samples were collected at 0, 1, 2, 3, 4, $6 \mathrm{w}$ after the administration, and the plasma was separated by centrifugation (12,000 rpm, $10 \mathrm{~min})$. The concentrations of triglycerides (TG), cholesterol, low-density lipoprotein (LDL) and high-density lipoprotein (HDL) in the plasma were quantitatively analyzed using the direct chemical determination method. In addition, after 6 weeks of administration, the rabbits were sacrificed. The mRNA level of peroxisome proliferator-activated receptor- $\gamma$ (PPAR- $\gamma$ ) and bone morphogenetic protein-2 (BMP-2) were determined by the method of fluorescence quantitative PCR (Fluorescence quantitative polymerase chain reaction, FQD96A, haier, Qingdao, China).

\section{Histomorphological Observation}

Six weeks after administration, the femoral head specimens were taken from animals in groups $(1,2,5)$, fixed with $4 \%$ formaldehydum polymerisatum, decalcified, embedded in paraffin and stained with routine HE. Osteogenesis in the femoral head, material degradation, morphological change and bone growth status were observed under an optical microscope and empty lacunae in five high-power fields of each specimen were counted.

\section{Statistical Analysis}

All data were presented as mean \pm SD. Statistical analysis was performed using the GraphPad Prism Version 4 software (GraphPad Software, Inc., La Jolla, CA, USA). Analysis of variance or the paired $t$ test was conducive to statistical analysis, and the statistical significance was set at $p<0.05$.

\section{Results and Discussion \\ Characterization}

The addition of an emulsifier was to prevent the aggregation of MS. ${ }^{18}$ As an emulsifier, PVA has been widely used in the preparation of MS. During the formation of MS, hydrophobic fragments of PVA permeated into the organic phase and remained in the polymer matrix of MS(PLGA). ${ }^{19}$ Then, by the way of steric hindrance, the hydrophilic fragments of PVA surrounded the system to stabilize the MS. ${ }^{20,21}$ In this study, we used the emulsionsolvent evaporation method to prepare LVTT-PLGA MS. Scanning electron microscopy demonstrated that the LVTT-PLGA MS were regular, round in shape and relatively unified size distributions were selected (Figure 1).

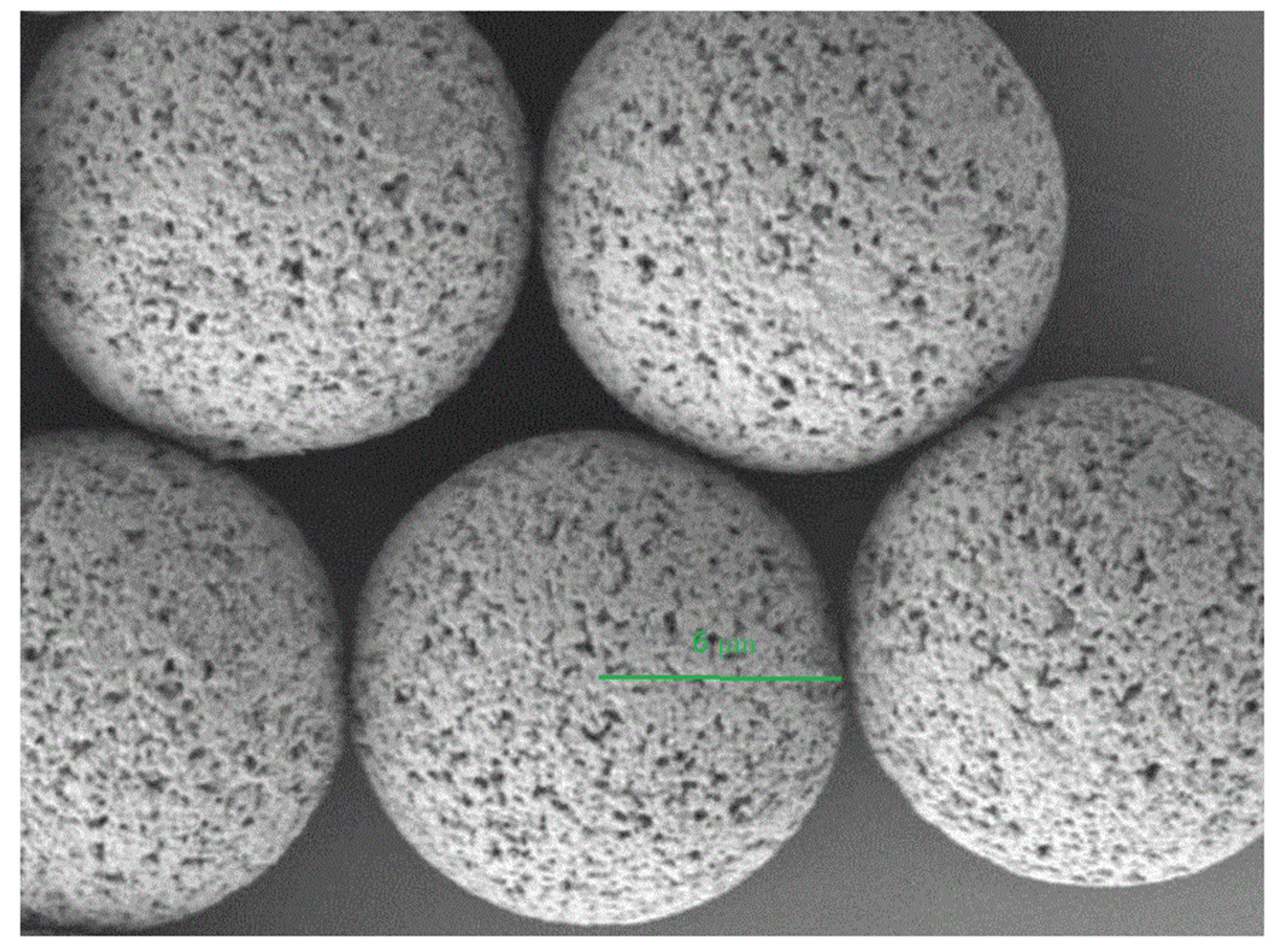

Figure I Scanning electron microscopy image of LVTT-PLGA MS. The green line represents the radius. Magnification $\times 5000$. 
Table 2 Physicochemical Properties of LVTT-PLGA MS $(n=3)$

\begin{tabular}{|l|l|l|l|l|l|}
\hline Formulations & $\begin{array}{l}\text { Particle Size } \\
(\boldsymbol{\mu m})\end{array}$ & $\begin{array}{l}\text { Encapsulation Efficiency } \\
(\%)\end{array}$ & $\begin{array}{l}\text { Drug Loading } \\
(\%)\end{array}$ & $\begin{array}{l}\text { Polydispersity } \\
\text { Index }\end{array}$ & $\begin{array}{l}\text { Zeta Potentials } \\
(\mathbf{m V})\end{array}$ \\
\hline LVTT-PLGA MS & $12.3 \pm 2.1$ & $87.3 \pm 5.6$ & $27.6 \pm 2.9$ & 0.21 & $-19.4 \pm 1.5$ \\
\hline
\end{tabular}

The mean PS was $12.3 \pm 2.1 \mu \mathrm{m}$. The drug-loading rate $(27.6 \% \pm 2.9 \%)$ was calculated for three batches of MS. The other parameters are shown in Table 2.

\section{DSC and XRD Analysis}

The interaction between the drug entity and the excipient in MS system was determined by the DSC method. Figure 2 shows the thermal behavior of the pure components and the final preparation (LVTT-PLGA MS). The LVTT peaks appeared clear, demonstrating a sharp characteristic endothermic peak at $174^{\circ} \mathrm{C}$ corresponding to its melting temperature (Figure 2A). The DSC thermal behaviors of the blank MS and physical mixture were chosen as a reference (Figure 2B and $\mathrm{C}$ ). The thermogram of LVTTPLGA MS showed an endothermic peak at $98.3^{\circ} \mathrm{C}$, revealing that LVTT existed in MS in an uncrystallized form rather than a crystallized one (Figure 2D).

The XRD study was carried out with the support of DSC to verify the reduction in crystalline nature of LVTT in prepared MS. The XRD spectrums of LVTT in Figure
3A and the physical mixture in Figure 3B showed distinct and intense peaks at $2 \theta$ scale, indicating the crystalline nature of the drug. The XRD spectrum of blank MS was chosen as a reference (Figure 3C). In contrast, there was a considerable decline in the intensity of all peaks in the XRD pattern of LVTT-PLGA MS as shown in Figure 3D. Therefore, it can be revealed that the LVTT drug may be in an amorphous state in the MS formulation.

\section{FTIR Analysis}

Figure 4 shows the FTIR spectra of LVTT, Blank MS, LVTT-PLGA MS. The LVTT spectrum displays the carbonyl $\mathrm{C}=\mathrm{O}$ stretching vibrations $\left(1700 \mathrm{~cm}^{-1}\right)$ and the $\mathrm{OH}$ stretching vibrations $\left(3541 \mathrm{~cm}^{-1}\right)$; the blank MS spectrum displays the stretching vibrations of PLGA ester group $\left(1754 \mathrm{~cm}^{-1}\right)$, the $\mathrm{OH}$ stretching vibrations $\left(3508 \mathrm{~cm}^{-1}\right)$. In the spectrum of LVTT-PLGA MS, in addition to the stretching vibration peak of the hydroxyl group and ester group of PLGA, the characteristic absorption peak of lactone carbonyl of the drug was also found at $1696 \mathrm{~cm}^{-1}$. These bands
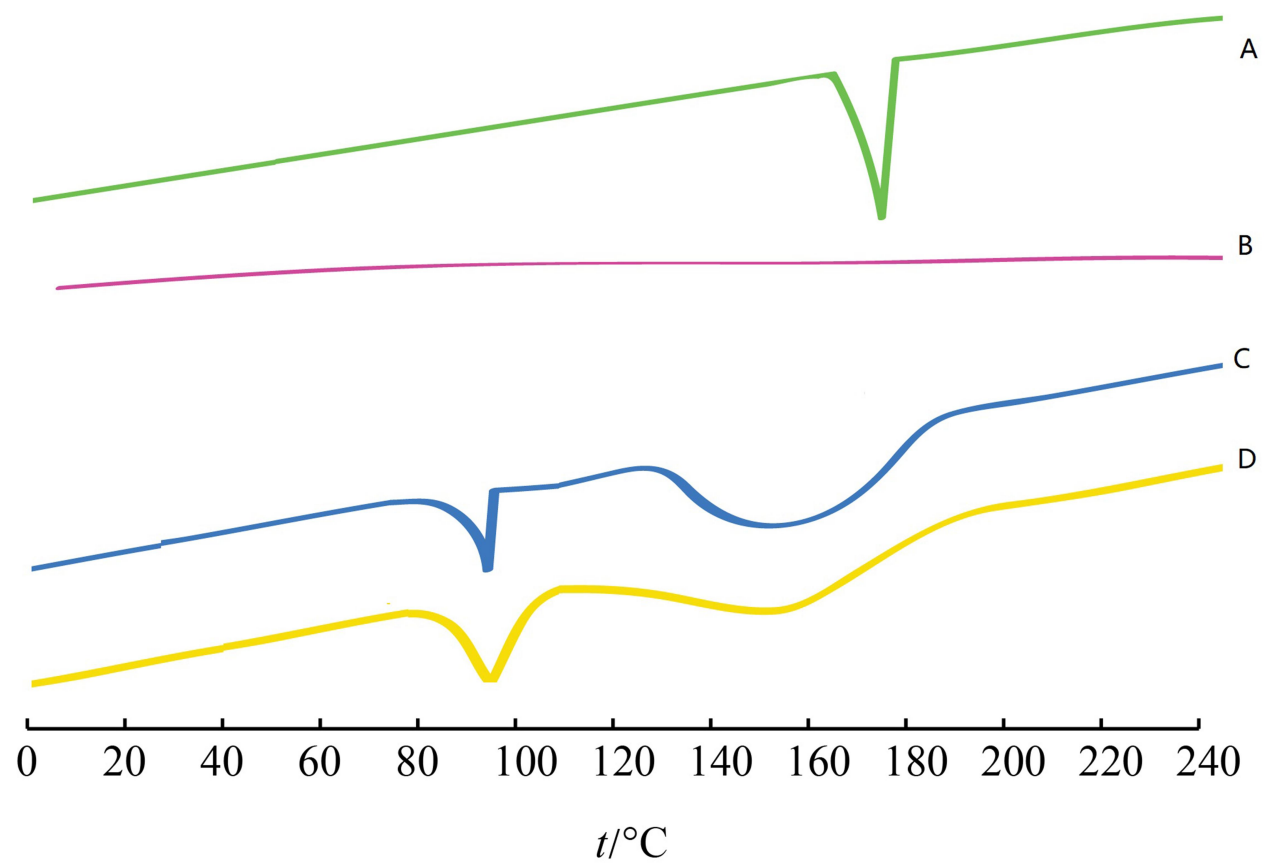

Figure 2 DSC analyses of the samples. (A) LVTT; (B) physical mixture of LVTT and blank MS; (C) blank MS; and (D) LVTT-PLGA MS. 


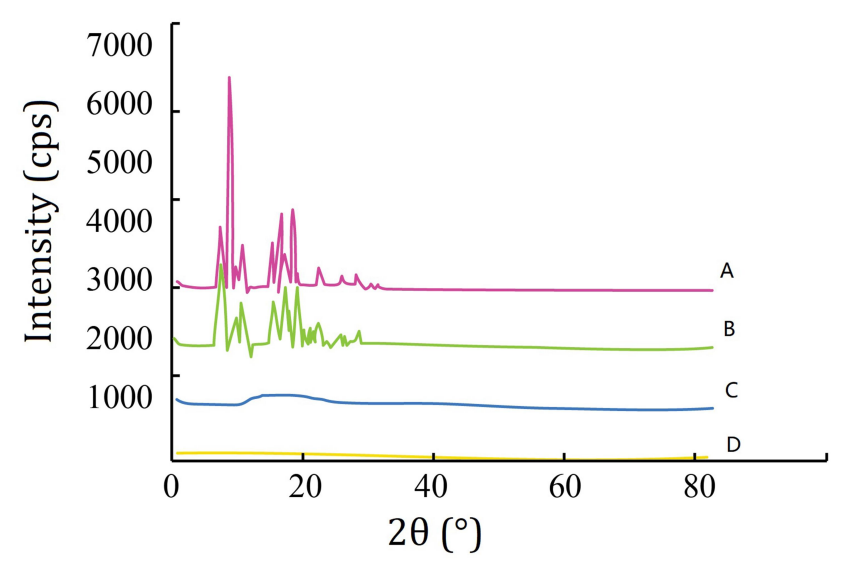

Figure 3 X-ray diffraction spectra. (A) LVTT; (B) physical mixture of LVTT and blank MS; (C) blank MS; and (D) LVTT-PLGA MS.

were distinct in the LVTT-PLGA MS; also, there were no major shifting, which confirmed the successful LVTT incorporation.

\section{In vitro Release Studies}

The in vitro release tests of LVTT-PLGA MS and free LVTT were carried out in PBS for 21 days, and their dissolution rates were determined under the same condition (Figure 5). On the first day, about 7\% of LVTT was released in MS, and more than 95\% of LVTT was dissolved in the free drug. Together with all the data obtained over the entire 21 days, it was found that the release rate of $\mathrm{MS}$ was much slower than that of the LVTT free drug. Drug release from biodegradable polymeric particles occurs through a combination of several mechanisms. It generally occurs through desorption of surface-bound drug, diffusion of the drug through the polymer matrix, and erosion of the polymer particles. $^{22,23}$ In this study, it is observed that there are linear prolonging drug release rates for more than 21 days without initial burst release.

We found that the release was about $30 \%$ for LVTTPLGA MS, which occurred within the first 5 days after maintaining the microspheres in PBS. The fact that there is no initial burst release suggests low drug density at the surface of the microspheres. This could happen due to the similar solubility of the polymer and the LVTT in the solvent, which means the drug was distributed among the MS quite homogenously. The rate of drug release gradually increased after about 16 days. The later constant release is mainly due to drug diffusion and matrix erosion mechanisms of biodegradable PLGA polymer. $^{24}$

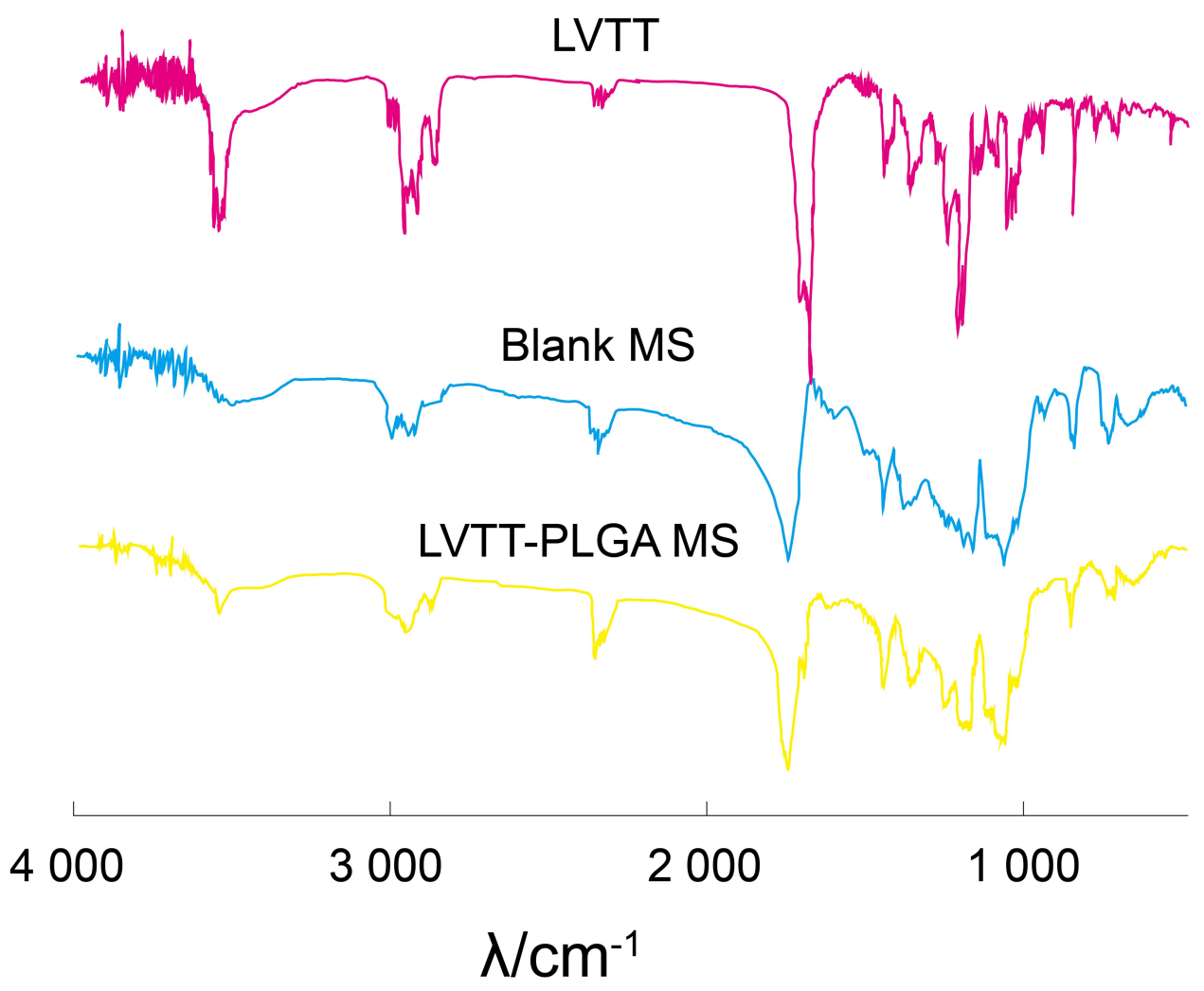

Figure 4 FTIR spectra of LVTT, blank MS and LVTT-PLGA MS. 


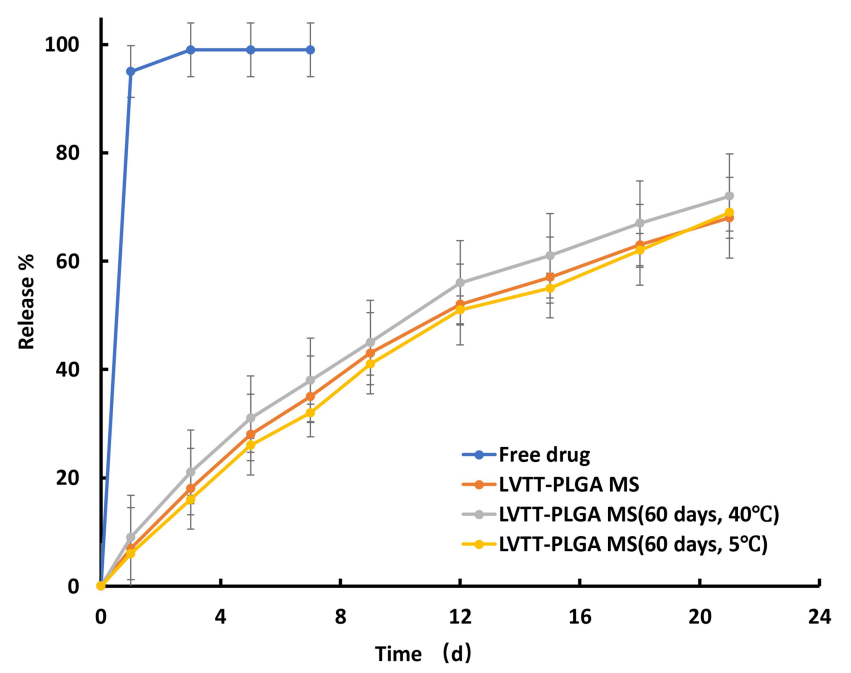

Figure $\mathbf{5}$ The release profile of free LVTT, LVTT-PLGA MS and LVTT-PLGA MS in PBS for 21 days $(n=6)$.

The in vitro release profile of LVTT from the MS was best expressed by a zero-order kinetic equation: $\ln$ $(1-\mathrm{Q})=-0.1718 \mathrm{t}+0.0625(\mathrm{R}=0.9967)$ in which $\mathrm{t}$ is the release time, $\mathrm{Q}$ is the percent of the drug released, and $\mathrm{R}$ is the correlation coefficient. This release was due to the diffusion and degradation of the microspheres.

\section{Stability Study}

Stability studies were performed under various temperatures in a period of 60 days. At regular intervals, the MS were subjected to drug content assay, and the results are shown in Table 3. At a higher temperature $\left(40^{\circ} \mathrm{C}\right)$, the percentage of encapsulation efficiency was found to be $83.1 \%$, whereas $85.5 \%$ was obtained at $5^{\circ} \mathrm{C}$ at the end of 60 days. There was no significant difference between them. At the same time, the storage period and condition had no significant effect on the particle size and drug loading. This result suggests that the MS were more stable at both temperatures. The in vitro release profiles of
LVTT-PLGA MS before and after stability studies are illustrated in Figure 4. The $f 2$ similarity factor was calculated by considering the release profile of the samples before the stability study as a reference and the release profile of samples after the stability study (60 days) as a test. The $f 2$ values were $66.9\left(40^{\circ} \mathrm{C}\right)$ and $87.3\left(5^{\circ} \mathrm{C}\right)$. These results confirmed the similarity of the release profiles of the formulation that was maintained at various temperatures for a storage period of 60 days.

\section{Pharmacodynamic Evaluation Biochemical Indexes}

The establishment of the animal model in this study was completely successful, and there were significant differences between the positive control group and the negative control group. Secondly, according to the situation of each group, the values of the three biochemical indexes (TG, cholesterol, LDL) showed different degrees of decline after different interventions. The intervention measures of group 3 did not significantly improve the indexes, while the intervention measures of group 4 and group 5 could significantly improve the three indexes, especially group 5 , which showed a relatively long-term effect. In HDL index, the trend of group 3, group 4 and group 5 was opposite. Group 5 could significantly and persistently improve HDL value (Figure 6). Similarly, at the end of the experimental observation period, we measured the PPAR- $\gamma$ and BMP-2 in rabbits and found that after local treatment, the value of group 5 was closer to that of the blank control group, and there were significant differences compared with the other two treatment groups (Figure 7).

The application of lipid-lowering drugs for the treatment and prevention of femoral head necrosis has become a hot spot in recent years. Relevant in vitro experiments have shown that statins can inhibit the effect of hormones, upregulate the expression of $\mathrm{Cbfa} 1$ (Growth rate compared to group 1: Group3 12\%;

Table 3 Physical and Chemical Properties and Stability Data of LVTT-PLGA MS. Data Represents the Mean \pm SD $(n=3)$

\begin{tabular}{|l|l|l|l|l|l|l|l|}
\hline Formulation & Temperature & Time & $\begin{array}{l}\text { Particle Size } \\
(\boldsymbol{\mu m})\end{array}$ & $\begin{array}{l}\text { Encapsulation } \\
\text { Efficiency }(\%)\end{array}$ & $\begin{array}{l}\text { Drug } \\
\text { Loading (\%) }\end{array}$ & $\begin{array}{l}\text { Polydispersity } \\
\text { Index }\end{array}$ & $\begin{array}{l}\text { Zeta Potentials } \\
(\mathbf{m V})\end{array}$ \\
\hline $\begin{array}{l}\text { LVTT-PLGA } \\
\text { MS }\end{array}$ & $\begin{array}{l}\text { Room } \\
\text { temperature }\end{array}$ & 0 day & $12.3 \pm 2.1$ & $87.3 \pm 5.6$ & $27.6 \pm 2.9$ & 0.21 & $-19.4 \pm 1.5$ \\
& $40 \pm 2^{\circ} \mathrm{C}$ & 30 days & $13.7 \pm 3.2$ & $85.2 \pm 7.2$ & $26.2 \pm 2.5$ & 0.23 & $-20.2 \pm 1.3$ \\
& 50 days & $14.5 \pm 3.3$ & $83.1 \pm 6.7$ & $25.1 \pm 2.3$ & 0.22 & $-21.3 \pm 1.6$ \\
& $5 \pm 2^{\circ} \mathrm{C}$ & 30 days & $12.6 \pm 2.3$ & $86.6 \pm 4.9$ & $26.7 \pm 2.3$ & 0.24 & $-22.1 \pm 1.4$ \\
& & 60 days & $12.8 \pm 2.1$ & $85.5 \pm 6.1$ & $26.2 \pm 2.2$ & 0.25 & $-22.5 \pm 1.7$ \\
\hline
\end{tabular}

Note: After statistics, there is no significant difference between the data of each group and the parameters of initial state ( 0 day). 

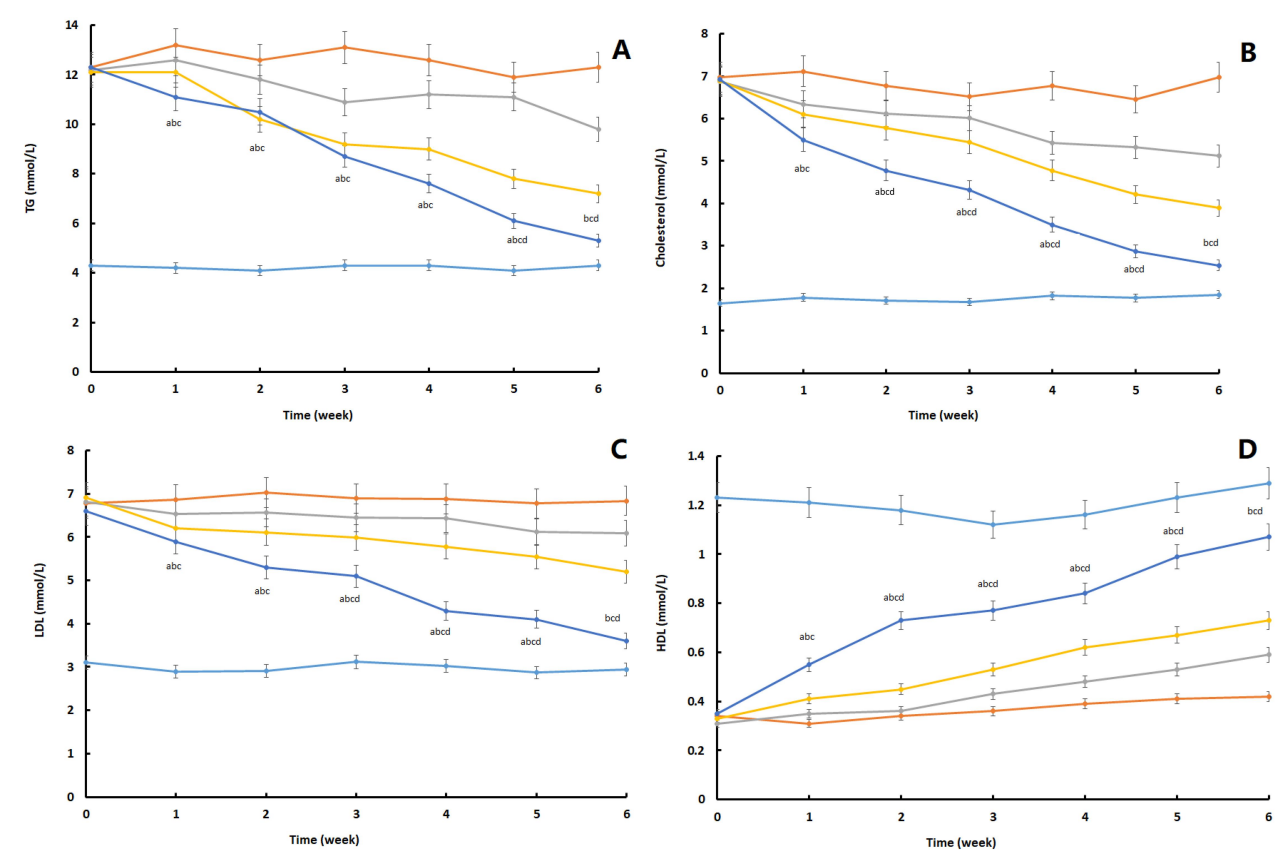

Figure 6 The change trend of blood lipid level in different experimental groups during the observation period. (A) TG; (B) cholesterol; (C) $L D L ;(D) ~ H D L ~(n=6) .{ }^{a} p<0.05$, Group 5 vs Group I; ${ }^{b} p<0.05$, Group 5 vs Group 2; ${ }^{c} p<0.05$, Group 5 vs Group 3; ${ }^{d}<<0.05$, Group 5 vs Group 4.

Group4 19\%; Group 26\%) and Runx2 (Growth rate compared to group 1: Group3 16\%; Group4 21\%; Group 29\%), and inhibit the expression of PPAR- $\gamma$, so as to prevent the fatty marrow cavity, reduce the intramedullary pressure, improve the local blood flow, reduce the cytotoxicity and increase the osteogenesis. $^{25,26}$ The current research results show that statins can prevent the necrosis of the femoral

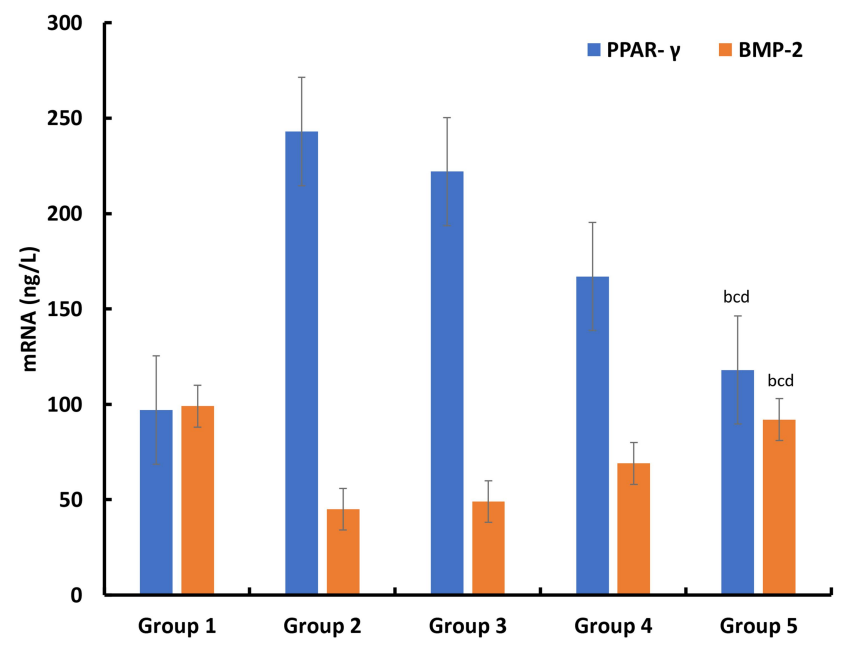

Figure 7 The concentration of PPAR- $\gamma$ and BMP-2 in rabbits after 6 weeks observation period $(n=6)$. ${ }^{b} p<0.05$, Group 5 vs Group 2 ; ${ }^{c} p<0.05$, Group 5 vs Group 3; ${ }^{d} p<0.05$, Group 5 vs Group 4. head and early treatment in animal models or in the human body with a large amount of hormone. However, due to necrosis of the femoral head, $85 \%$ of statins are metabolized in the liver, and only $5 \%$ of the remaining $15 \%$ exist in the blood in a free state, so that few drugs reach the local area. In this way, it is impossible to achieve the concentration of statins that has been proposed in recent years to induce osteogenesis and reduce adiposis, which also leads to the poor effect of conservative treatment of avascular necrosis of the femoral head.

Based on the understanding of osteonecrosis of the femoral head, local delivery of drug is worthy of research. In the above study, the therapeutic value of LVTT-PLGA MS on femoral head necrosis was analyzed. PLGA is a kind of biodegradable polymer materials, which can be used as drug-delivery system to combine with LVTT for local injection. First of all, this study compared the blood lipid metabolism of three groups of rabbits with femoral head necrosis after treatment. The results showed that local injection of LVTT could significantly improve the whole-body lipid metabolism, especially MS. At present, it has been found that hormone can induce bone marrow stromal cells to upregulate PPAP- $\gamma$ and downregulate BMP-2, thus leading to the occurrence of 

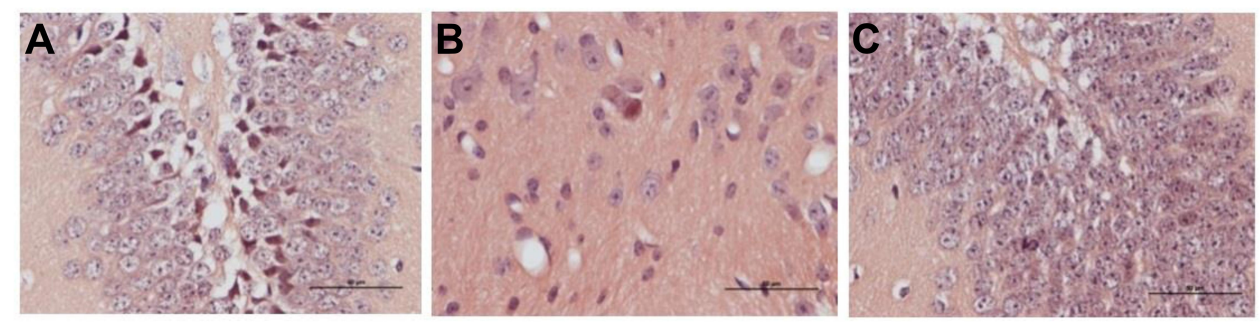

Figure 8 Histological observations on rabbit femoral head in three groups 6 weeks after operation (HE staining, ×40). (A) Group I; (B) Group 2; (C) Group 5 $($ Bar $=100 \mu \mathrm{m})$.

femoral head necrosis. ${ }^{27}$ Therefore, this study through the detection of these two indexes to reflect the situation of the femoral head. From the experimental results, the improvement of MS group is the most obvious.

\section{Histomorphological Observation}

Six weeks after administration, histological observations were performed in the three groups. Group 2: Blank control group, the cartilage layer became thinner and the necrotic chondrocytes arranged orderly. Group 5: articular cartilage and subchondral bone were thickened and chondrocytes were arranged orderly. Adipocytes are rare. The trabeculae were densely distributed and showed brittleness. Empty bone lacunae in trabecular bone were rare, and the number of empty bone lacunae in group 5 was significantly less than that in group $2(\mathrm{P}<0.05)$. There are a lot of osteoblasts around trabeculae (Figure 8).

\section{Conclusions}

In this study, we used the emulsion-solvent evaporation method to prepare LVTT-PLGA MS. Scanning electron microscopy demonstrated that the LVTT-PLGA MS were regular, round in shape and relatively unified size distributions were selected. The mean PS was $12.3 \pm 2.1 \mu \mathrm{m}$. The drug-loading rate $(27.6 \% \pm 2.9 \%)$ was calculated for three batches of MS. The thermogram of LVTT-PLGA MS showed an endothermic peak at $98.3^{\circ} \mathrm{C}$, revealing that LVTT existed in MS in an uncrystallized rather than a crystallized form. In the release study, LVTT-PLGA MS is observed with linear prolonging drug release rates for more than 21 days without initial burst release. The pharmacodynamic results confirmed that the LVTT-PLGA MS had a good and lasting improvement effect against femoral head necrosis compared with the common injection. Our results demonstrated that LVTT-PLGA MS has the potential for being a local delivery system.

\section{Disclosure}

The authors report no conflicts of interest in this work.

\section{References}

1. Yoshii T, Hafeman AE, Esparza JM, Okawa A, Gutierrez G, Guelcher SA. Local injection of lovastatin in biodegradable polyurethane scaffolds enhances bone regeneration in a critical-sized segmental defect in rat femora. J Tissue Eng Regen Med. 2014;8 (8):589-595. doi:10.1002/term.1547

2. Murray SS, Tu KN, Young KL, Murray EJB. The effects of lovastatin on proteasome activities in highly purified rabbit $20 \mathrm{~S}$ proteasome preparations and mouse MC3T3-E1 osteoblastic cells. Metabolism. 2002;51(9):1153-1160. doi:10.1053/meta.2002.34706

3. Ghosh-Choudhury N, Mandal CC, Choudhury GG. Statin-induced Ras activation integrates the phosphatidylinositol 3-kinase signal to Akt and MAPK for bone morphogenetic protein-2 expression in osteoblast differentiation. J Biol Chem. 2007;282(7):4983-4993. doi:10.1074/jbc.M606706200

4. Maeda T, Matsunuma A, Kurahashi I, Yanagawa T, Yoshida H, Horiuchi N. Induction of osteoblast differentiation indices by statins in MC3T3-E1 cells. J Cell Biochem. 2004;92(3):458-471. doi:10.10 02/jcb. 20074

5. Wong RWK, Rabie AB. Early healing pattern of statin-induced osteogenesis. Br J Oral Maxillofac Surg. 2005;43(1):46-50. doi:10.1016/j.bjoms.2004.08.014

6. Weis M, Heeschen C, Glassford AJ, Cooke JP. Statins have biphasic effects on angiogenesis. Circulation. 2002;105(6):739-745. doi:10.11 61/hc0602.103393

7. Choi K-Y, Kim H-J, Lee M-H, et al. Runx2 regulates FGF2-inducedBmp2 expression during cranial bone development. Dev Dyn. 2005;233(1):115-121. doi:10.1002/dvdy.20323

8. Bouletreau PJ, Warren SM, Spector JA, et al. Hypoxia and VEGF Up-Regulate BMP-2 mRNA and Protein Expression in Microvascular Endothelial Cells: implications for Fracture Healing. Plast Reconstr Surg. 2002;109(7):2384-2397. doi:10.1097/00006 534-200206000-00033

9. Rao S, Tan A, Boyd BJ, Prestidge CA. Synergistic role of self-emulsifying lipids and nanostructured porous silica particles in optimizing the oral delivery of lovastatin. Nanomedicine. 2014;9 (18):2745-2759. doi:10.2217/nnm.14.37

10. Lennernäs H, Fager G. Pharmacodynamics and pharmacokinetics of the HMG-CoA reductase inhibitors. Similarities and differences. Clin Pharmacokinet. 1997;32(5):403-425. doi:10.2165/00003088-19973 2050-00005

11. Chen C-H, Uang Y-S, Wang S-T, Yang J-C, Lin C-J. Interaction between red yeast rice and CYP450 enzymes/P-glycoprotein and Its implication for the clinical pharmacokinetics of lovastatin. Evid Based Complement Alternat Med. 2012;2012:127043. doi:10.1155/ 2012/127043 
12. Sun JX, Niecestro R, Phillips G, Shen J, Lukacsko P, Friedhoff L. Comparative pharmacokinetics of lovastatin extended-release tablets and lovastatin immediate-release tablets in humans. $J$ Clin Pharmacol. 2002;42(2):198-204. doi:10.1177/00912700222011111

13. Steinbach JM, Seo Y-E, Saltzman WM. Cell penetrating peptide-modified poly(lactic-co-glycolic acid) nanoparticles with enhanced cell internalization. Acta Biomater. 2016;30:49-61. doi:10.1016/j.actbio.2015.11.029

14. Zhang C, An T, Wang D, et al. Stepwise $\mathrm{pH}$-responsive nanoparticles containing charge-reversible pullulan-based shells and poly $(\beta$-amino ester)/poly(lactic-co-glycolic acid) cores as carriers of anticancer drugs for combination therapy on hepatocellular carcinoma. $J$ Control Release. 2016;226:193-204. doi:10.1016/j.jconrel.20 16.02 .030

15. Guan Q, Chen W, Hu X. Development of lovastatin-loaded poly (lactic acid) microspheres for sustained oral delivery: in vitro and ex vivo evaluation. Drug Des Devel Ther. 2015;9:791-798. doi:10.2147/DDDT.S76676

16. Moore JW, Flanner HH. Mathematical comparison of dissolution profiles. Pharm Tech. 1996;20:64-75.

17. Tian L, Dang X-Q, Wang C-S, Yang P, Zhang C, Wang K-Z. Effects of sodium ferulate on preventing steroid-induced femoral head osteonecrosis in rabbits. J Zhejiang Univ Sci B. 2013;14(5):426-437. doi:10.1631/jzus.B1200311

18. Castellanos IJ, Flores G, Griebenow K. Effect of the molecular weight of poly(ethylene glycol) used as emulsifier on $\alpha$ chymotrypsin stability upon encapsulation in PLGA microspheres. J Pharm Pharmacol. 2005;57(10):1261-1269. doi:10.1211/jpp.57. 10.0004

19. Li G, Yao L, Li J, Qin X, Qiu Z, Chen W. Preparation of poly (lactide-co-glycolide) microspheres and evaluation of pharmacokinetics and tissue distribution of BDMC-PLGA-MS in rats. Asian J Pharm Sci. 2018;13(1):82-90. doi:10.1016/j.ajps.2017.09.002
20. Gu B, Sun X, Papadimitrakopoulos F, Burgess DJ. Seeing is believing, PLGA microsphere degradation revealed in PLGA microsphere/ PVA hydrogel composites. J Control Release. 2016;228:170-178. doi:10.1016/j.jconrel.2016.03.011

21. Freiberg S, Zhu XX. Polymer microspheres for controlled drug release. Int J Pharm. 2004;282(1-2):1-18. doi:10.1016/j.ijpharm.20 04.04.013

22. Malipeddi VR, Awasthi R, Dua K. Formulation and evaluation of controlled release ethylcellulose and polyethylene glycol microspheres containing metoprolol tartrate.. Intervent Med App Sci. 2016;8(2):60-67. doi:10.1556/1646.8.2016.2.6

23. Ahnfelt E, Sjögren E, Hansson P, Lennernas H. In Vitro Release Mechanisms of Doxorubicin From a Clinical Bead Drug-Delivery System. J Pharm Sci. 2016;105(11):3387-3398. doi:10.1016/j.xphs. 2016.08.011

24. Mahboubian A, Hashemein SK, Moghadam S, Atyabia F, Dinarvand R. Preparation and In-vitro Evaluation of Controlled Release PLGA Microparticles Containing Triptoreline.. Iran J Pharm Res. 2010;9(4):369-378.

25. Rajamannan NM, Subramaniam M, Springett M, et al. Atorvastatin inhibits hypercholesterolemia-induced cellular proliferation and bone matrix production in the rabbit aortic valve. Circulation. 2002;105 (22):2660-2665. doi:10.1161/01.CIR.0000017435.87463.72

26. Hong W, Wei Z, Qiu Z, et al. Atorvastatin promotes bone formation in aged apoE-/- mice through the Sirt1-Runx2 axis. J Orthop Surg Res. 2020;15(1):303. doi:10.1186/s13018-020-01841-0

27. Nishida K, Yamamoto $T$, Motomura G, Jingushi S, Iwamoto $Y$. Pitavastatin may reduce risk of steroid-induced osteonecrosis in rabbits: a preliminary histological study. Clin Orthop Relat Res. 2008;466(5):1054-1058. doi:10.1007/s11999-008-0189-4

\section{Publish your work in this journal}

Drug Design, Development and Therapy is an international, peerreviewed open-access journal that spans the spectrum of drug design and development through to clinical applications. Clinical outcomes, patient safety, and programs for the development and effective, safe, and sustained use of medicines are a feature of the journal, which has also been accepted for indexing on PubMed Central. The manuscript management system is completely online and includes a very quick and fair peer-review system, which is all easy to use. Visit http://www. dovepress.com/testimonials.php to read real quotes from published authors. 